\title{
Hypertranscendance des systèmes aux différences diagonaux
}

\author{
Charlotte Hardouin
}

\begin{abstract}
This paper deals with criteria of algebraic independence for the derivatives of solutions of diagonal difference systems. The key idea consists in deriving from the commutativity of the differentiation and difference operators a sequence of iterated extensions of the original difference module, thereby setting the problem in the framework of difference Galois theory and finally reducing it to an exercise in linear algebra on the group of divisors of the involved elliptic curve or torus.
\end{abstract}

\section{RÉSUMÉ}

On donne dans cet article divers critères d'indépendance algébrique pour les dérivées successives de solutions de systèmes aux différences diagonaux. L'idée principale consiste à construire au moyen de l'opérateur de dérivation, qui commute avec l'opérateur aux différences, des extensions itérées du module aux différences initial. Le problème se ramène alors au calcul du groupe de Galois aux différences de telles extensions, calcul qui lui-même se réduit à une simple question d'algèbre linéaire sur le groupe des diviseurs de la courbe elliptique ou du tore mis en jeu.

\section{Introduction}

\section{1 Énoncé des résultats}

Dans cet article, on s'intéresse aux relations algébro-différentielles satisfaites par les solutions d'équations fonctionnelles, plus particulièrement aux différences. On dira qu'une fonction $f$ (indéfiniment dérivable) est hypertranscendante sur un corps $K$ s'il n'existe pas de relations algébriques à coefficients dans $K$ liant $f$ et ses dérivées, autrement dit si $f$ ne vérifie pas d'équation différentielle algébrique sur $K$.

L'exemple le plus classique de fonction hypertranscendante est celui de la fonction $\Gamma$ qui vérifie l'équation fonctionnelle $\Gamma(z+1)=z \Gamma(z)$ et qui est hypertranscendante sur $\mathbb{C}(z)$. Des résultats de même nature ont été établis par exemple par Bank (voir [Ban80]) dans le cadre d'équations aux $\tau$-différences de rang 1 et par Ishizaki (voir [Ish98]) dans le cadre d'équations aux $q$-différences non homogènes de rang 1. Ces résultats se basent essentiellement sur des méthodes analytiques et sur des estimations de la taille des coefficients du développement de telles fonctions, telles qu'étudiées dans [Ram92].

Dans cet article, on cherche à étendre ces énoncés à l'étude simultanée de plusieurs solutions (autrement dit, à des questions d'hyperindépendance algébrique), et ce, par des méthodes purement algébriques de théorie de Galois aux différences. Voici, dans le cadre des $q$-différences, le type de théorème auquel on aboutira.

Received 20 September 2007, accepted in final form 26 November 2007.

2000 Mathematics Subject Classification 12H10, 12H05, 34M15, 39A13.

Keywords: linear difference equations, difference Galois groups, tannakian category, hypertranscendence.

This journal is (C) Foundation Compositio Mathematica 2008. 


\section{HARDOUin}

Soit $q \in \mathbb{C}^{*},|q| \neq 1$. On désigne par $\operatorname{Mer}\left(\mathbb{C}^{*}\right)$ le corps des fonctions méromorphes sur $\mathbb{C}^{*}$, par $\sigma_{q}$ l'automorphisme de $\mathcal{M e r}\left(\mathbb{C}^{*}\right)$, qui à une fonction $f(z)$ associe la fonction $f(q z)$ et par $C_{E}$ le sous-corps de $\operatorname{Mer}\left(\mathbb{C}^{*}\right)$ des fonctions invariantes sous l'action de $\sigma_{q}$, qui est isomorphe au corps $\mathbb{C}(E)$ des fonctions rationnelles sur la courbe elliptique $E=\mathbb{C}^{*} / q^{\mathbb{Z}}$. On appelle diviseur elliptique d'une fonction $f \in \mathbb{C}(z)$ l'image dans le groupe des diviseurs de la courbe elliptique $E=\mathbb{C}^{*} / q^{\mathbb{Z}}$ de la partie première à $\{0, \infty\}$ du diviseur de $f$.

ThÉorème 1.1. Soient $a_{1}, \ldots, a_{n}$ des éléments non nuls de $\mathbb{C}(z)$ et $q$ un nombre complexe non nul de module différent de 1 . Pour tout $i=1, \ldots, n$, soit $f_{i} \neq 0$ une solution méromorphe sur $\mathbb{C}^{*}$ de l'équation aux q-différences $\sigma_{q}\left(f_{i}\right)=a_{i} f_{i}$. On suppose que les diviseurs elliptiques des $a_{i}$ sont linéairement indépendants sur $\mathbb{Z}$. Alors les fonctions $f_{1}, \ldots, f_{n}$ ainsi que leur dérivées successives sont algébriquement indépendantes $\operatorname{sur} C_{E}(z)$.

On obtient un énoncé similaire pour les $\tau$-différences, qui entraîne, par exemple, que les seules relations de dépendance algébriques liant les fonctions polygamma $\psi^{(k)}(z+\alpha), \psi^{(k)}(n z), k, n \in \mathbb{N}$, $\alpha \in \mathbb{C}$ se déduisent de la relation de distribution satisfaite par la fonction gamma.

\subsection{Principe de la preuve}

L'outil essentiel de la démonstration du Théorème 1.1 est la théorie de Galois aux différences, qui permet de faire le lien entre groupe de Galois des équations et degré de transcendance des extensions de corps mises en jeu (tout comme en théorie des équations différentielles). Le principe de la preuve est le suivant.

Soit $(K, \sigma, \partial)$ un corps aux différences et différentiel de caractéristique nulle, c'est à dire un corps $K$ muni d'un automorphisme de corps $\sigma$ et d'un opérateur de dérivation $\partial$ tels que $\sigma \circ \partial=\partial \circ \sigma$. On note $C_{\sigma}$ (respectivement $C_{\partial}$ ) le sous-corps de $K$ formé des invariants sous $\sigma$ (respectivement des constantes différentielles de $K$ ) et $\mathcal{D}_{K}=K\left[\sigma, \sigma^{-1}\right]$ l'anneau des polynômes en $\sigma$ et $\sigma^{-1}$ à coefficients dans $K$. Par exemple, avec les notations du $\S 1.1$, on pourra prendre $(K, \sigma, \partial)=\left(C_{E}(z), \sigma_{q}, z d / d z\right)$ (d'où $C_{\sigma}=C_{E}$ et $C_{\partial}=\mathbb{C}$ ), ou encore $(K, \sigma, \partial)=\left(\mathbb{C}(z), \sigma_{q}, z d / d z\right)$ (d'où $C_{\sigma}=C_{\partial}=\mathbb{C}$ ).

On considère le système aux différences

$$
\sigma Y=A Y, \text { où } A \in G l_{n}(K) .
$$

Soient $F$ une extension aux différences différentielle de $K$ et $Y \in F^{n}$ une solution de (1). Notons $\mathcal{A}$ le $\mathcal{D}_{K}$-module associé à ce système et considérons le vecteur

$$
\left(\begin{array}{c}
\partial Y \\
Y
\end{array}\right) \in F^{2 n}
$$

Puisque $\sigma$ et $\partial$ commutent, il vérifie l'équation aux différences

$$
\sigma\left(\begin{array}{c}
\partial Y \\
Y
\end{array}\right)=\left(\begin{array}{cc}
A & \partial A \\
0 & A
\end{array}\right)\left(\begin{array}{c}
\partial Y \\
Y
\end{array}\right)
$$

qui correspond à une extension $\tilde{\mathcal{M}}(1)$ de $\mathcal{A}$ par $\mathcal{A}$ dans la catégorie des $\mathcal{D}_{K}$-modules.

En itérant $N-1$ fois ce processus, et en notant que pour tout entier $j \geqslant 1$,

$$
\sigma\left(\partial^{j} Y\right)=\partial^{j}(\sigma Y)=\partial^{j}(A Y)=\sum_{i=0}^{j} C_{j}^{i} \partial^{i} A \partial^{j-i} Y
$$




\section{HyPERTRANSCENDANCE DES SYSTÈMES AUX DIFFÉRENCES DIAGONAUX}

on obtient le système aux différences

$$
\sigma\left(\begin{array}{c}
\partial^{N} Y \\
\cdot \\
\cdot \\
\cdot \\
\cdot \\
\cdot \\
\cdot \\
\cdot \\
\partial Y \\
Y
\end{array}\right)=\left(\begin{array}{ccccccc}
A & \ldots & \ldots & C_{N}^{k} \partial^{k} A & \cdots & C_{N}^{1} \partial^{N-1} A & \partial^{N} A \\
0 & A & \ldots & \vdots & \ldots & \ldots & \vdots \\
0 & \ldots & \ddots & C_{N-r}^{k-r} \partial^{k-r} A & \ldots & \ldots & \partial^{N-r} A \\
0 & \ldots & \ldots & \ldots & \ldots & \ldots & \vdots \\
0 & \ldots & \ldots & A & \ldots & \ldots & \vdots \\
0 & \ldots & \ldots & \ldots & \ddots & \ldots & \partial^{2} A \\
0 & \ldots & \ldots & \ldots & \ldots & A & \partial A \\
0 & \ldots & \ldots & \ldots & \ldots & 0 & A
\end{array}\right)\left(\begin{array}{c}
\partial^{N} Y \\
\cdot \\
\cdot \\
\cdot \\
\cdot \\
\cdot \\
\cdot \\
\partial \\
. \\
\end{array}\right)
$$

C'est la représentation matricielle d'un $\mathcal{D}_{K}$-module $\tilde{\mathcal{M}}(N)$, extension itérée $N$-fois de l'objet $\mathcal{A}$ dans cette catégorie.

Le degré de transcendance du corps $K\left(Y, \partial Y, \ldots, \partial^{N} Y\right)$ sur $K$ s'interprète alors comme la dimension de l'orbite de cette solution sous l'action du groupe de Galois aux différences $G_{\tilde{\mathcal{M}}(N)}$ de $\tilde{\mathcal{M}}(N)$. De plus, lorsque $\mathcal{A}$ est complétement réductible la seule action du radical unipotent de $G_{\tilde{\mathcal{M}}(N)}$ suffit à contrôler le degré de transcendance de la partie différentielle $K\left(Y, \partial Y, \ldots, \partial^{N} Y\right) / K(Y)$ de cette extension.

On ne sait, en toute généralité, déterminer ces radicaux unipotents. Toutefois, dans le cas d'une somme directe $\mathcal{A}$ de $\mathcal{D}_{K}$-modules de rang 1 comme au Théorème 1.1 , le calcul du radical unipotent de $G_{\tilde{\mathcal{M}}(N)}$ se simplifie (voir Remarque 3.1). Sous ses hypothèses, on verra qu'il est aussi grand que possible. Le Théorème 1.1 et son analogue pour les $\tau$-différences en découlent immédiatement.

\subsection{Choix d'un foncteur fibre et plan de l'article}

Pour calculer les groupes de Galois, on doit disposer d'une catégorie tannakienne et en choisir un foncteur fibre. Par exemple, dans le cas des q-différences, celui des symboles de van der Put et Singer [vdPS97], où la catégorie tannakienne $\mathbf{T}$ est la catégorie des $\sigma_{q}$-modules de type fini sur $K=\mathbb{C}(z)$ (ou plus généralement sur $K=C(z)$ avec $C$ algébriquement clos de caractéristique nulle), est un foncteur fibre $\omega$ sur $\mathbb{C}$ (ou $C$; voir aussi [And01, paragraphes 3.2 et 3.4]). Les foncteurs fibres, de nature plus géométrique, de Sauloy ([Sau04]) fournissent encore des groupes de Galois sur $\mathbb{C}$. Les travaux de Praagman [Pra86] permettent en revanche de considérer la catégorie $\mathbf{T}^{\prime}=\mathbf{T}_{\mathbf{E}}$ des $\sigma_{q}$-modules de type fini sur $K_{E}=C_{E}(z)$ et un foncteur fibre $\omega_{E}$ (donc un groupe de Galois) sur le corps $C_{E}$. La nature même de nos résultats (étude de 'vraies' fonctions, sur lesquelles agit une dérivation) conduit à privilégier cette dernière approche. On doit néanmoins prendre garde au fait que le corps des constantes $C^{\prime}=C_{E} n^{\prime}$ est alors plus algébriquement clos.

$\mathrm{Au} \S 2$, on calcule ainsi le groupe de Galois d'extensions de l'objet unité $\mathbf{1}$ de $\mathbf{T}^{\prime}$ par lui-même. Il est unipotent, et le résultat obtenu est un analogue du théorème d'Ostrowski. On calcule de même le groupe de Galois d'une somme directe $\mathcal{A}$ de $\mathcal{D}_{K}$-modules de rang 1 (analogue du théorème de Kolchin). On rappelle par ailleurs comment le groupe de Galois contrôle le degré de transcendance des solutions. La Proposition 2.7 résume ce dont nous aurons besoin par la suite.

Dans le $\S 3$, on étudie les extensions de la catégorie $\mathbf{T}_{\mathbf{E}}$ mises en jeu au Théorème 1.1. Leur indépendance se ramène à une simple question d'algèbre linéaire, qui permet d'aboutir aux hypothèses sur les diviseurs elliptiques du Théorème 1.1 (voir Lemme 3.8), et à sa conclusion.

Enfin, on donne dans le $\S 4$ l'analogue des théorèmes de le $\S 3$ dans le cadre des $\tau$-différences.

On trouvera en appendice une autre preuve du Théorème 1.1, due à van der Put [vdP07]. Elle a pour cadre la catégorie $\mathbf{T}$ de [vdPS97], la catégorie $\mathbf{T}_{\mathbf{E}}$ n'apparaissant qu'au tout dernier point. 


\section{HARDOUin}

On retrouvera dans ce cadre un analogue de la Proposition 2.7, et une preuve plus concise des critères d'indépendance linéaire du $\S 3$.

Remarque 1.2. La considération simultanée de deux opérateurs, ici $\sigma_{q}$ et $\partial$, agissant sur le même corps de fonctions n'est pas nouvelle, voir par exemple les travaux de Bézivin [Béz00] pour deux opérateurs aux $q$-différences, $\sigma_{q_{1}}$ et $\sigma_{q_{2}}$ avec $q_{1} \neq q_{2}$. Dans ce type de travail, les deux opérateurs jouent un rôle symétrique et on ne traite que d'équations linéaires en chacun des opérateurs.

L'idée clé du présent article est la considération des extensions successives $\tilde{\mathcal{M}}(N)$ décrites plus haut. Les rôles des opérateurs $\sigma_{q}$ et $\partial$ ne sont plus symétriques, permettant ainsi d'atteindre des équations différentielles non linéaires. Signalons que ce processus s'adapte à d'autres situations: voir ainsi le recent travail de Ovchinnikov [Ovc06], où $\sigma_{q}$ est remplacé par un operateur de derivation $\partial_{x}$ commutant avec $\partial_{z}$.

Remarque 1.3. La comparaison entre les groupes de Galois de van der Put et Singer et ceux attachés au foncteur fibre de Praagman est traitée dans [CHS08], où il est démontré que ces groupes deviennent isomorphes sur la clôture algébrique de $C_{E}{ }^{1}$

\section{Calcul de groupes de Galois et changement de base}

Dans toute cette partie, on fixe un corps aux différences $\left(K^{\prime}, \sigma\right)$ de corps des constantes $C^{\prime}$ de caractéristique nulle. On ne suppose pas $C^{\prime}$ algébriquement clos. On désigne par $\mathcal{D}_{K^{\prime}}=K^{\prime}\left[\sigma, \sigma^{-1}\right]$ l'anneau des opérateurs polynomiaux en $\sigma$ et $\sigma^{-1}$ à coefficients dans $K^{\prime}$ et par $\mathbf{T}^{\prime}=\operatorname{Diff}\left(\mathbf{K}^{\prime}, \sigma\right)$ la catégorie des $\mathcal{D}_{K^{\prime}}$-modules de dimension finie sur $K^{\prime}$. On suppose donné un foncteur fibre $\omega^{\prime}$ de $\operatorname{Diff}\left(K^{\prime}, \sigma\right)$ dans la catégorie $\operatorname{Vect}_{C^{\prime}}$ des espaces vectoriels de dimension finie sur $C^{\prime}$ et on note $G$ le groupe proalgébrique attaché à ce foncteur. La catégorie tannakienne neutre $\operatorname{Diff}\left(K^{\prime}, \sigma\right)$ est alors équivalente à la catégorie $R e p_{G / C^{\prime}}$ des représentations de $G$ sur les objets de $\operatorname{Vect}_{C^{\prime}}$.

Soit $\mathcal{M}$ un objet de $\operatorname{Diff}\left(K^{\prime}, \sigma\right)$. On désigne par $\langle\mathcal{M}\rangle$ la sous-catégorie tannakienne engendrée par $\mathcal{M}$ dans $\operatorname{Diff}\left(K^{\prime}, \sigma\right)$ et par $G_{\mathcal{M}}=A u t^{\otimes}\left(\omega^{\prime} \mid\langle\mathcal{M}\rangle\right)$ son groupe de Galois: c'est un groupe algébrique sur $C^{\prime}$, image de $G$ par la représentation $\rho_{\mathcal{M}}$ de $G$ que découpe $\mathcal{M}$.

On note $\operatorname{Ext}_{\sigma}^{1}(\mathbf{1}, \mathbf{1})$ (respectivement $\left.\operatorname{Ext}_{\operatorname{Rep}_{G / C^{\prime}}}\left(C^{\prime}, C^{\prime}\right)\right)$ le groupe des extensions de l'objet trivial 1 (respectivement $C^{\prime}$ ) par lui-même dans la catégorie $\operatorname{Diff}\left(K^{\prime}, \sigma\right)$ (respectivement $R e p_{G / C^{\prime}}$ ). Ces groupes sont naturellement munis de structures d'espaces vectoriels sur le corps $C^{\prime}=\operatorname{End}_{D i f f\left(K^{\prime}, \sigma\right)}$ $(\mathbf{1})=\operatorname{End}_{\operatorname{Rep}_{G / C^{\prime}}}\left(C^{\prime}\right)$.

\subsection{Un analogue des théorèmes d'Ostrowski et de Kolchin}

Proposition 2.1 ('Théorème d'Ostrowski'). Soient $\mathcal{E}_{1}, \ldots, \mathcal{E}_{n}$ des extensions de $\mathbf{1}$ par $\mathbf{1}$ dans la catégorie Diff $\left(K^{\prime}, \sigma\right)$. Soient $b_{1}, \ldots, b_{n}$ des éléments de $K$ tels que pour tout $i=1, \ldots, n, \mathcal{E}_{i}$ admettent une représentation matricielle donnée par

$$
\left(\begin{array}{cc}
1 & b_{i} \\
0 & 1
\end{array}\right)
$$

Alors $G_{\mathcal{E}_{1} \oplus \cdots \oplus \mathcal{E}_{n}}=\left(\mathbf{G}_{a / C^{\prime}}\right)^{n}$ si et seulement si $b_{1}, \ldots, b_{n}$ sont $C^{\prime}$-linéairement indépendants modulo $(\sigma-\mathrm{id})\left(K^{\prime}\right)$.

\footnotetext{
${ }^{1}$ Le présent article est une version allégée de [Har06], où le comportement des objets de $\mathbf{T}$ par changement de base est étudié plus en détail, et où cette comparaison est effectuée dans le cas unipotent.
} 


\section{HYPERTRANSCENDANCE DES SYSTÈMES AUX DIFFÉRENCES DIAGONAUX}

Démonstration. Soit $\mathcal{E} \in \operatorname{Ext} t_{\sigma}^{1}(\mathbf{1}, \mathbf{1})$. La représentation $\rho_{\mathcal{E}}$ de $G$ decoupée par $\mathcal{E}$ est un objet de $\operatorname{Ext}_{R e p_{G / C^{\prime}}}^{1}\left(C^{\prime}, C^{\prime}\right)$, donc de la forme

$$
\rho_{\mathcal{E}}=\left(\begin{array}{cc}
1 & f_{\mathcal{E}} \\
0 & 1
\end{array}\right),
$$

où $f_{\mathcal{E}}$ est un homomorphisme défini sur $C^{\prime}$ du groupe proalgébrique $G$ dans le groupe additif $\mathbf{G}_{a}$, et on déduit de l'équivalence des catégories $\operatorname{Diff}(K, \sigma)$ et $\operatorname{Rep}_{G / C^{\prime}}$ que l'application $f: \mathcal{E} \mapsto f_{\mathcal{E}}$ ainsi définie est un isomorphisme $C^{\prime}$-linéaire de $\operatorname{Ext} t_{\sigma}^{1}(\mathbf{1}, \mathbf{1})$ dans $\operatorname{Hom}\left(G, \mathbf{G}_{a} / C^{\prime}\right)$. Le groupe de Galois $G_{\mathcal{E}}$ de $\mathcal{E}$ est dans ce cas isomorphe a $f_{\mathcal{E}}(G)$.

Plus généralement, posons $\mathcal{M}:=\bigoplus_{i=1}^{n} \mathcal{E}_{i}$ et notons $f_{i}:=f_{\mathcal{E}_{i}}$ le morphisme attaché a $\mathcal{E}_{i}$ pour tout $i=1, \ldots, n$. Le groupe de Galois $G_{\mathcal{M}}$ de $\mathcal{M}$ est alors isomorphe à l'image de $G$ par $\left(f_{1}, \ldots, f_{n}\right)$ : $G \rightarrow \mathbf{G}_{a}^{n}$. Si $G_{\mathcal{E}_{1} \oplus \cdots \oplus \mathcal{E}_{n}} \subsetneq \mathbf{G}_{a}^{n}$, on déduit de la structure des sous-groupes algébriques propres de $\mathbf{G}_{a}^{n}$ en caractéristique 0 qu'il existe $\lambda_{1}, \ldots, \lambda_{n} \in C^{\prime}$ non tous nuls tels $\lambda_{1} f_{1}+\cdots+\lambda_{n} f_{n}=0$. Posons $\mathcal{E}=\lambda_{1} \mathcal{E}_{1}+\cdots+\lambda_{n} \mathcal{E}_{n}$ dans $\operatorname{Ext}_{\sigma}^{1}(\mathbf{1}, \mathbf{1})$. Par $C^{\prime}$-linéarité de $f$, on a $f_{\mathcal{E}}=\lambda_{1} f_{\mathcal{E}_{1}}+\cdots+\lambda_{n} f_{\mathcal{E}_{n}}=0$, et on déduit de l'injectivité de $f$ que l'extension $\mathcal{E} \in \operatorname{Ext}_{\sigma}^{1}(\mathbf{1}, \mathbf{1})$ est alors triviale.

Or l'extension $\mathcal{E}$ de $\mathbf{1}$ par $\mathbf{1}$ ainsi définie admet pour représentation matricielle

$$
\left(\begin{array}{ll}
1 & b \\
0 & 1
\end{array}\right)
$$

où $b=\lambda_{1} b_{1}+\cdots+\lambda_{n} b_{n}$, et une telle extension est triviale si et seulement si $b \in(\sigma-1)\left(K^{\prime}\right)$. Ainsi, $G_{\mathcal{E}_{1} \oplus \cdots \oplus \mathcal{E}_{n}} \subsetneq C^{\prime n}$ si et seulement si $b_{1}, \ldots, b_{n}$ sont $C^{\prime}$-linéairement dépendants modulo $(\sigma-$ id) $\left(K^{\prime}\right)$.

Proposition 2.2 ('Théorème de Kolchin'). Soient $a_{1}, \ldots, a_{n}$ des éléments de $K^{*}$, et $\mathcal{A}$ le $\mathcal{D}_{K^{\prime}}$ module de rang $n$ associé au système

$$
\sigma Y=\left(\begin{array}{cccc}
a_{1} & 0 & \cdots & 0 \\
0 & a_{2} & \cdots & 0 \\
\vdots & 0 & \ddots & \vdots \\
0 & \cdots & 0 & a_{n}
\end{array}\right) Y:=A Y
$$

Alors, le groupe de Galois $G_{\mathcal{A}}$ de $\mathcal{A}$ est un sous-groupe strict de $\left(\mathbf{G}_{m} / C^{\prime}\right)^{n}$ si et seulement il existe des entiers $r_{1}, \ldots r_{n}$ non tous nuls, et un élément $h$ de $K^{*}$ tels que $\prod_{i} a_{i}^{r_{i}}=\sigma_{q}(h) / h$.

Démonstration. Pour tout $i=1, \ldots, n$, notons $\mathcal{A}_{i}$ le $\mathcal{D}_{K^{\prime}}$-module associé à l'équation $\sigma_{q}(y)=a_{i} y$, de sorte que $\mathcal{A}=\bigoplus_{i} \mathcal{A}_{i}$, et $\rho: G \rightarrow\left(\mathbf{G}_{m / C^{\prime}}\right)^{n}$ la représentation de $G$ découpée par $\mathcal{A}$. Alors, $\rho(G)=G_{\mathcal{A}}$ est un sous-groupe propre de $\left(\mathbf{G}_{m}\right)^{n}$ si et seulement s'il est annulé par un caractère $\chi$ de $\left(\mathbf{G}_{m}\right)^{n}$ non trivial, c'est-à-dire si et seulement s'il existe des entiers $r_{1}, \ldots, r_{n}$ non tous nuls (décrivant le caractère $\chi(x)=x_{1}^{r_{1}} \ldots x_{n}^{r_{n}}$ ), tels que

$$
\chi \circ \rho(G) \simeq G_{\otimes_{i} \mathcal{A}_{i}{ }^{\otimes r_{i}}}=\{1\}
$$

Cela revient à dire que le $\mathcal{D}_{K^{\prime}}$-module $\bigotimes_{i} \mathcal{A}_{i}{ }^{\otimes r_{i}}$ est trivial, ou encore qu'il existe un élément $h$ de $K^{\prime}$ tel que

$$
a_{1}^{r_{1}} \cdots a_{n}^{r_{n}}=\frac{\sigma(h)}{h} .
$$

\subsection{Lien avec la transcendance}

On rappelle qu'on ne suppose pas $C^{\prime}$ algébriquement clos.

Proposition 2.3. Soit $\left(K^{\prime}, \sigma\right)$ un corps aux différences de corps des constantes $C^{\prime}$ de caractéristique nulle. On suppose donnée une extension $F$ de corps aux différences de $K^{\prime}$, de même corps des 


\section{HARDOUin}

constantes $C^{\prime}$, et vérifiant la propriété suivante: tout système aux $\sigma$-différences à coefficients dans $K^{\prime}$ admet une matrice fondamentale de solutions à coefficients dans $F$. On note $\omega^{\prime}$ le foncteur fibre de Diff $\left(K^{\prime}, \sigma\right)$ à valeurs dans Vect $_{C^{\prime}}$ correspondant.

Soient $\mathcal{M}$ un objet de Diff $\left(K^{\prime}, \sigma\right)$, et $U$ une matrice fondamentale de solutions à coefficients dans $F$. Alors, le groupe de Galois $G_{\mathcal{M}}$ de $\mathcal{M}$ relativement à $\omega^{\prime}$ vérifie

$$
\operatorname{deg} \operatorname{tr}\left(K^{\prime}(U) / K^{\prime}\right)=\operatorname{dim}_{C^{\prime}} G_{\mathcal{M}} .
$$

Démonstration. La preuve qui suit étend la démonstration de [Kat90, p. 38] au cas d'un corps de constantes non algébriquement clos.

Comme plus haut, notons $\langle\mathcal{M}\rangle$ la catégorie tannakienne engendrée par $\mathcal{M}$ dans $\operatorname{Diff}\left(K^{\prime}, \sigma\right)$, de sorte que $G_{\mathcal{M}}$ est le $C^{\prime}$-groupe algébrique $A u t^{\otimes}\left(\omega^{\prime} \mid\langle\mathcal{M}\rangle\right)$. Soient $M \subset \mathcal{M} \otimes_{K^{\prime}} F$ le $C^{\prime}$-espace vectoriel $\omega^{\prime}(\mathcal{M})$, et $v$ un élément de $M\left(C^{\prime}\right)$. Alors, $M$ est une $C^{\prime}$-représentation de $G_{\mathcal{M}}$, et l'image schématique $G_{\mathcal{M}}(v)$ du morphisme de $G_{\mathcal{M}}$ dans $M$ attaché à $v$ est un $C^{\prime}$-sous-schéma de $M$ (voir [Spr98, 1.9.1 (iv)]). L'annulateur de $v \otimes 1$ dans le $K^{\prime}$-dual $\mathcal{M}^{*}$ de $\mathcal{M}$ est un sous-objet $\mathcal{W}$ de $\mathcal{M}^{*}$ dans la catégorie $\langle\mathcal{M}\rangle$, et $W:=\omega^{\prime}(\mathcal{W})$ est un sous- $G_{\mathcal{M}}$-module du $C^{\prime}$-dual $M^{*}$ de $M$. Comme $W \subset \mathcal{W} \otimes_{K^{\prime}} F$ annule $v$ et est $G_{\mathcal{M}}$-stable, il doit annuler $G_{\mathcal{M}}(v)$. Ainsi, $W$ est contenu dans l'annulateur $S$ de $G_{\mathcal{M}}(v)$ dans $V^{*}$. Comme $S$ est stable sous $G_{\mathcal{M}}$, il lui correspond par équivalence de catégories un sous-objet $\mathcal{S}$ de $\mathcal{M}^{*}$ dans la catégorie $\langle\mathcal{M}\rangle$. Comme $S$ annule $v$, il en est de même de $\mathcal{S} \subset S \otimes_{C^{\prime}} F$, et on a $\mathcal{S} \subset \mathcal{W}$. Mais $W \subset S$, donc $\mathcal{S}=\mathcal{W}$. Ainsi, la dimension sur $C^{\prime}$ de l'espace des formes linéaires sur $V$ annulant $G_{\mathcal{M}}(v)$ est égale à la dimension sur $K^{\prime}$ de l'espace des formes linéaires sur $\mathcal{M}$ qui annulent $v \otimes 1$.

En appliquant ce résultat au vecteur $\bigoplus_{i \leqslant n} \operatorname{Symm}^{i}(v)$ et à l'objet $\bigoplus_{i \leqslant n} \operatorname{Symm}^{i}(\mathcal{M})$ de $\langle\mathcal{M}\rangle$, on obtient que le degré de transcendance de $K^{\prime}(v)$ sur $K^{\prime}$ est égale à la dimension sur $C^{\prime}$ de $G_{\mathcal{M}}(v)$.

Enfin, appliquons ce dernier résultat à l'objet $\mathcal{M}^{\prime}:=\underline{\operatorname{Hom}}\left(M \otimes K^{\prime}, \mathcal{M}\right)$ de $\langle\mathcal{M}\rangle$. Sa fibre $\omega^{\prime}\left(\mathcal{M}^{\prime}\right)$ s'identifie à $\operatorname{End}(M)$ et $G_{\mathcal{M}}$ y agit par translation à gauche. La matrice fondamentale de solutions $U$ de $\mathcal{M}$ fournit un élément de $\omega^{\prime}\left(\mathcal{M}^{\prime}\right)$ dans $G L(M)\left(C^{\prime}\right)$. Comme $G_{\mathcal{M}}$ agit librement sur $G L(M) / C^{\prime}$, on en déduit que

$$
\operatorname{deg} \operatorname{tr}\left(K^{\prime}(U) / K^{\prime}\right)=\operatorname{dim}_{C^{\prime}} G_{\mathcal{M}}
$$

\subsection{Extension des scalaires}

Soit $K$ un sous-corps de $K^{\prime}$ stable sous $\sigma$, et $C$ le corps des constantes de $K$. On se propose de descendre de $K^{\prime}$ à $K$ les hypothèses faites aux Propositions 2.1 et 2.2. On trouvera dans [Har0] et [Har06] des conditions générales le permettant. Nous nous contenterons ici d'énoncer un resultat.

Lemme 2.4. On suppose qu'il existe un élément $z$ de $K^{\prime}$, transcendant sur $C^{\prime}$, tel que $K=C(z)$ et $K^{\prime}=C^{\prime}(z)$. Le corps $C$ est supposé algébriquement clos. Soient $b_{1}, \ldots, b_{n}$ des éléments de $K$. Alors, $b_{1}, \ldots, b_{n}$ sont $C^{\prime}$-linéairement indépendants modulo $(\sigma-\mathrm{id})\left(K^{\prime}\right)$ si et seulement si $b_{1}, \ldots, b_{n}$ sont $C$-linéairement indépendants modulo $(\sigma-\mathrm{id})(K)$.

Démonstration. L'une des implications est évidente. Réciproquement, supposons que $b_{1}, \ldots, b_{n}$ sont $C^{\prime}$-linéairement dépendants modulo $(\sigma-\mathrm{id})\left(K^{\prime}\right)$, et soit

$$
\sum_{j=1}^{n} \lambda_{i} b_{i}=\sigma(g)-g
$$

une relation de dépendance non triviale, avec $\lambda_{1}=1$. Soit $d \in C^{\prime}[z]$ un dénominateur de $g$, et $R$ la $C$-sous-algèbre de $C^{\prime}$ engendrée par les $\lambda_{i}$ et les coefficients de $d$ et de $d g=a$. Comme $R$ est de type fini sur $C$, il existe un homomorphisme de spécialisation $\pi: R \rightarrow C$ tel que $\pi(d) \in C[z]$ soit 


\section{HyPERTRANSCENDANCE DES SYSTÈMES AUX DIFFÉRENCES DIAGONAUX}

non nul. Alors, $\sigma(g)=(a / d)(\sigma(z))$, où $\sigma(z)=(\alpha z+\beta) /(\gamma z+\delta)$ pour une matrice

$$
\left(\begin{array}{ll}
\alpha & \beta \\
\gamma & \delta
\end{array}\right) \in P G L_{2}(C)
$$

et la fonction rationnelle $\bar{g}=\pi(a) / \pi(d) \in C(z)=K$ vérifie

$$
\sum_{j=1}^{n} \pi\left(\lambda_{i}\right) b_{i}=\sigma(\bar{g})-\bar{g} .
$$

Comme $\pi\left(\lambda_{1}\right)=1$, les $b_{i}$ sont donc $C$-linéairement dépendants modulo $(\sigma-1)(K)$.

Le même argument de spécialisation entraîne, de façon encore plus directe.

Lemme 2.5. On reprend les hypothèses sur $K$ et $K^{\prime}$ du Lemme 2.4. Soient $a_{1}, \ldots, a_{n}$ des éléments de $K^{*}$. On suppose qu'il existe des entiers $r_{1}, \ldots r_{n}$ non tous nuls, et un élément $h^{\prime}$ de $K^{\prime *}$ tels que $\prod_{i} a_{i}^{r_{i}}=\sigma_{q}\left(h^{\prime}\right) / h^{\prime}$. Alors, il existe un élément $h$ de $K^{*}$ tels que $\prod_{i} a_{i}^{r_{i}}=\sigma_{q}(h) / h$.

Remarque 2.6. Pour tout objet $\mathcal{M}$ de la catégorie $\operatorname{Diff}(K, \sigma)$, notons $\mathcal{M}^{\prime}=\mathcal{M} \otimes_{K} K^{\prime}$ l'objet de $\operatorname{Diff}\left(K^{\prime}, \sigma\right)$ déduit de $\mathcal{M}$ par extension des scalaires de $K$ à $K^{\prime}$. Le Lemme 2.5 exprime qu'en rang 1 , $\mathcal{M}^{\prime}$ ne peut être trivial que si $\mathcal{M}$ l'est déjà, le Lemme 2.4 que les images dans $\operatorname{Diff}\left(K^{\prime}, \sigma\right)$ d'éléments de $\operatorname{Ext}_{\operatorname{Diff}(K, \sigma)}(\mathbf{1}, \mathbf{1})$ linéairement indépendants sur $C$ le restent sur $C^{\prime}$. Comme on l'a dit plus haut, ces résultats restent valables sous des hypothèses plus générales sur l'extension $K^{\prime} / K$ et s'étendent à tout objet $\mathcal{M}$; voir [Har06].

\subsection{Conclusion}

On se place dans le cadre du $\S 2.3$, avec $K=C(z), K^{\prime}=C^{\prime}(z)$, et on suppose donnée une extension $F$ de $K^{\prime}$ vérifiant les hypothèses de la Proposition 2.3. De plus, on fixe une dérivation $\partial$ de $K^{\prime}$ commutant avec $\sigma$ et laissant stable $K$.

Proposition 2.7. Soient $a_{1}, \ldots, a_{n}$ des éléments de $K^{*}$ et $N$ un entier naturel positif. On considère une solution $\left\{f_{i}, g_{i, j}, i=1, \ldots, n, j=0, \ldots, N-1\right\}$ dans $F^{n(N+1)}$, avec $f_{i} \neq 0$ pour tout $i$, du sytème d'équations aux différences

$$
\sigma\left(Y_{i}\right)=a_{i} Y_{i}, \quad \sigma\left(Z_{i, j}\right)=Z_{i, j}+\partial^{m}\left(\frac{\partial a_{i}}{a_{i}}\right) ; \quad i=1, \ldots, n, j=0, \ldots, N-1 .
$$

Supposons que les $n N$ éléments $\partial^{j}\left(\partial a_{i} / a_{i}\right),(i=1, \ldots, n ; j=0, \ldots, N-1)$, de $K$ sont $C$-linéairement indépendants modulo $(\sigma-1)(K)$. Alors, le degré de transcendance du corps $K^{\prime}\left(\left\{f_{i}, g_{i, j}, i=1, \ldots, n\right.\right.$, $j=0, \ldots, N-1\})$ sur $K^{\prime}$ est égal à $n(N+1)$.

Démonstration. Montrons tout d'abord que sous cette hypothèse, les $a_{i}$ sont multiplicativement indépendants modulo le sous-groupe $\left\{\sigma(h) / h, h \in K^{*}\right\}$ de $K^{*}$. Dans le cas contraire, on aurait une relation non triviale $a^{r_{1}} \cdots a_{n}^{r_{n}}=\sigma(h) / h$, d'où par dérivation logarithmique: $r_{1} \partial a_{1} / a_{1}+\cdots+$ $r_{n} \partial a_{n} / a_{n}=\partial \sigma h / \sigma h-\partial h / h=\sigma(\partial h / h)-\partial h / h$, et les $\partial a_{i} / a_{i}$ seraient $C$-linéairement dépendants modulo $(\sigma-1)(K)$.

Dans ces conditions, soit $\mathcal{M}(N)$ le $\mathcal{D}_{K^{\prime}}$-module associé au système $(*)$. On a: $\mathcal{M}(N)=\bigoplus_{i=1}^{n} \mathcal{A}_{i} \oplus$ $\bigoplus_{i=1, \ldots, n}^{j=0, \ldots, N} \mathcal{E}_{i, j}$ où pour tout $i=1, \ldots, n$ et $j=0, \ldots, N-1, \mathcal{A}_{i}$ représente le $\mathcal{D}_{K^{\prime}}$-module associé au système $\sigma\left(Y_{i}\right)=a_{i} Y_{i}$ et $\mathcal{E}_{i, j}$ est l'extension de $\mathbf{1}$ par $\mathbf{1}$ dans $\operatorname{Diff}\left(K^{\prime}, \sigma\right)$ associée au système $\sigma\left(Z_{i, j}\right)=Z_{i, j}+\partial^{j}\left(\partial a_{i} / a_{i}\right)$. De la semisimplicité de $G_{\bigoplus_{i=1}^{n} \mathcal{A}_{i}}$ et de l'unipotence de $G_{\bigoplus_{i=1, \ldots, n}^{j=0, \ldots, 1} \mathcal{E}_{i, j}}$, on déduit que

$$
G_{\mathcal{M}(N)}=G_{\bigoplus_{i=1}^{n} \mathcal{A}_{i}} \times G_{\bigoplus_{i=1, \ldots, n}^{j=0, \ldots, N-1} \mathcal{E}_{i, j}}
$$




\section{HARDOUin}

Les Propositions 2.1 et 2.2, jointes aux Lemmes 2.4 et 2.5, entraînent que ces facteurs sont respectivement isomorphes à $\mathbf{G}_{m}^{n}$ et à $\mathbf{G}_{a}^{n N}$. La dimension de $G_{\mathcal{M}(N)}$ vaut donc $n+n N$. Comme la matrice fondamentale $U$ du système associé à $\mathcal{M}(N)$ est la matrice bloc-diagonale constituée des $f_{i}$, non nuls, et des matrices

$$
\left(\begin{array}{cc}
1 & g_{i, j} \\
0 & 1
\end{array}\right)
$$

la Proposition 2.3 permet de conclure.

\section{Hypertranscendance des solutions d'équations aux $q$-différences}

\subsection{Méthode de démonstration du Théorème 1.1}

Comme dans l'introduction, soit $q \in \mathbb{C}^{*}$ un nombre complexe de module différent de 1 . Soient $F=\operatorname{Mer}\left(\mathbb{C}^{*}\right)$ le corps des fonctions méromorphes sur $\mathbb{C}^{*}$ et $\sigma_{q}$ l'automorphisme de $F$ qui à $f(z) \in F$ associe $f(q z)$. Soient $C_{E}$ l'ensemble des fonctions de $F$ fixées par $\sigma_{q}$, et $K_{E}=C_{E}(z)$ le compositum de $C_{E}$ et de $K=\mathbb{C}(z)$ dans $F$. Les corps $F$ et $K_{E}$ sont des corps aux différences relativement à $\sigma_{q}$, ayant le même corps de $\sigma_{q}$-constantes $C_{E}$, et $C_{E}$ s'identifie au corps des fonctions rationnelles sur la courbe elliptique $E=\mathbb{C}^{*} / q^{\mathbb{Z}}$. Les hypothèses du Lemme 2.4 sont alors vérifiées avec

$$
C=\mathbb{C}, \quad C^{\prime}=C_{E}, \quad K=\mathbb{C}(z), \quad K^{\prime}=K_{E}=C_{E}(z) .
$$

Dans [Pra86], Praagman démontre que toute équation aux q-différences à coefficients méromorphes sur $\mathbb{C}^{*}$ admet une $C_{E^{-}}$base de solutions méromorphes sur $\mathbb{C}^{*}$. En particulier, l'extension $F / K_{E}$ vérifie les hypothèses de la Proposition 2.3, et on dispose ainsi d'un foncteur fibre $\omega_{E}$ de $\operatorname{Diff}\left(K_{E}, \sigma_{q}\right)$ à valeur dans $\operatorname{Vect}_{C_{E}}$, qui attache à tout module aux $q$-différences sur $K_{E}$ le $C_{E^{-}}$ espace vectoriel de ses solutions dans $F=\operatorname{Mer}\left(\mathbb{C}^{*}\right)$, et qui munit $\mathbf{T}_{\mathbf{E}}=\operatorname{Diff}\left(\mathbf{K}_{\mathbf{E}}, \sigma_{\mathbf{q}}\right)$ d'une structure de catégorie tannakienne neutre sur le corps non algébriquement clos $C_{E}$. Comme on l'a dit au début de l'article, c'est ce foncteur fibre que nous privilégions, car il conduit naturellement à des extensions de corps aux différences différentiels: la dérivation $\partial=z d / d z$ de $F$ laisse stables $K_{E}$ et $K$, et commute à $\sigma_{q}$. Les hypothèses de la Proposition 2.7 sont donc satisfaites. Les groupes de Galois aux différences du $\S 2$ sur lesquels reposent notre preuve sont ainsi relatifs à $\omega_{E}$, et sont donc des groupes algébriques sur $C_{E}$.

On se place sous les hypothèses du Théorème 1.1, dont on reprend les notations. Des relations $\sigma_{q}\left(f_{i}\right)=a_{i} f_{i}, i=1, \ldots, n$, on déduit comme à la formule (2) que $\sigma_{q}\left(\partial f_{i}\right)=a_{i} \partial f_{i}+\partial a_{i} f_{i}$, de sorte que la dérivée logarithmique $Z_{i, 0}=\partial f_{i} / f_{i}$ de $f_{i}$ vérifie l'équation inhomogène

$$
\sigma_{q}\left(\frac{\partial f_{i}}{f_{i}}\right)=\frac{\partial f_{i}}{f_{i}}+\frac{\partial a_{i}}{a_{i}}
$$

qui correspond à une extension de l'objet unité par lui même. En dérivant à nouveau cette identité, on voit que pour tout $i=1, \ldots, n, j=0, \ldots, N-1$, la dérivée $\partial^{j}\left(\partial f_{i} / f_{i}\right)$ vérifie l'équation

$$
\sigma_{q}\left(Z_{i, j}\right)=Z_{i, j}+\partial^{j}\left(\frac{\partial a_{i}}{a_{i}}\right) .
$$

On est donc dans les conditions de la Proposition 2.7. Puisque les corps $K_{E}\left(f_{i}, \partial^{j}\left(\partial f_{i} / f_{i}\right)\right.$, $i=1, \ldots, n, j=0, \ldots, N-1)$ et $K_{E}\left(\partial^{j} f_{i}, i=1, \ldots, n, j=0, \ldots, N\right)$ coïncident, celle-ci entraîne que

$$
\operatorname{degtr}_{K_{E}}\left(K_{E}\left(\partial^{j}\left(f_{i}\right) ; i=1, \ldots, n, j=0, \ldots, N\right)\right)=n(N+1),
$$

autrement dit que ces $n(N+1)$ fonctions sont, pour tout entier $N$, algébriquement indépendantes sur $K_{E}$, sous réserve que les éléments $\partial^{j}\left(\partial a_{i} / a_{i}\right)(i=1, \ldots, n ; j=0, \ldots, N-1)$ de $K$ soient $C$-linéairement indépendants modulo $\left(\sigma_{q}-1\right)(K)$. 


\section{HyPERTRANSCENDANCE DES SYSTÈMES AUX DIFFÉRENCES DIAGONAUX}

Pour établir le Théorème 1.1, il reste donc à traduire cette dernière condition en termes de diviseurs elliptiques. C'est l'objet $\mathrm{du} \S 3.3$ (Lemme 3.8) ci-dessous.

Remarque 3.1. La méthode décrite dans l'introduction de l'article est ici simplifiée. La raison en est que pour une somme directe $\mathcal{A}$ d'objets $\mathcal{A}_{i}$ de rang 1, l'existence d'un isomorphisme entre $\operatorname{Ext}_{\text {Diff }(K, \sigma)}^{1}\left(\mathcal{A}_{i}, \mathcal{A}_{i}\right)$ et $\operatorname{Ext}_{\text {Diff }(K, \sigma)}^{1}(\mathbf{1}, \mathbf{1})$ permet de remplacer l'extension itérée $\tilde{\mathcal{M}}(N)$ du $\S 1.2$ par la somme directe $\mathcal{M}(N)$ de l'objet $\mathcal{A}$ et des extensions simples $\mathcal{E}_{i, j}$ intervenant dans la preuve de la Proposition 2.7. ${ }^{2}$

\subsection{Diviseurs elliptiques}

On rappelle que $K=\mathbb{C}(z)$, et que $E$ désigne la courbe elliptique $\mathbb{C}^{*} / q^{\mathbb{Z}}$.

DÉfinition 3.2. Soit $a \in K^{*}=\mathbb{C}(z)^{*}$. On dira que $a$ est standard ([vdPS97, ch. 2]) si, pour tout $\alpha \in \mathbb{C}^{*}$ dans le support du diviseur de $a$, et tout entier $m$ non nul, $q^{m} \alpha$ n'apparaît pas dans le diviseur de $a$.

Lemme 3.3. Soit a un élément de $K^{*}$. Il existe un couple $(g, \bar{a})$, avec $g \in K^{*}$ et $\bar{a}$ standard, tel que $a=\bar{a} \sigma_{q}(g) / g$. Une telle décomposition est dite forme standard de $a$.

Démonstration. Voir [vdPS97, Lemmes 2.1 et 2.2], où ce lemme est établi dans le cadre des $\tau$-différences. La démonstration dans le cas des $q$-diférences est entièrement analogue.

DÉfinition 3.4. Soient $a \in \mathbb{C}(z)^{*}$. On appelle diviseur elliptique de $a$ l'image $\operatorname{div}_{E}(a)$ de la partie première à $\{0, \infty\}$ du diviseur de $a$ par l'application naturelle de $\operatorname{Div}\left(\mathbb{C}^{*}\right)$ dans $\operatorname{Div}(E)=$ $\operatorname{Div}\left(\mathbb{C}^{*} / q^{\mathbb{Z}}\right)$.

Autrement dit, si $a(z)=\lambda z^{r} \prod_{\alpha \in \mathbb{C}^{*}}(z-\alpha)^{n_{\alpha}}$, on a $\operatorname{div}_{E}(a)=\sum_{\bar{\alpha} \in \mathbb{C}^{*} / q^{\mathbb{Z}}}\left(\sum_{\alpha \in \bar{\alpha}} n_{\alpha}\right) \cdot(\bar{\alpha})$. En écrivant $a$ sous forme standard (Lemme 3.3), on en déduit Lemme 3.5.

Lemme 3.5. Soient $a \in K^{*}$. Alors $\operatorname{div}_{E}(a)=0$ si et seulement s'il existe un entier $r$, un nombre complexe $\mu \in \mathbb{C}^{*}$ et un élément $h$ de $K^{*}$ tel que $a=\mu z^{r} \sigma_{q}(h) / h$.

\subsection{Indépendance de diviseurs}

Nous pouvons maintenant terminer la démontration du Théorème 1.1 de l'introduction, dont nous rappelons ici l'énoncé sous une forme plus précise.

ThÉorème 3.6. Soient $a_{1}, \ldots, a_{n}$ des éléments non nuls de $K=\mathbb{C}(z)$ et $q$ un nombre complexe non nul de module différent de 1 . Pour tout $i=1, \ldots, n$, soit $f_{i} \neq 0$ une solution méromorphe sur $\mathbb{C}^{*}$ de l'équation aux q-différences $\sigma_{q}\left(f_{i}\right)=a_{i} f_{i}$. Alors:

(i) les fonctions $f_{1}, \ldots, f_{n}$ sont algébriquement dépendantes sur $K_{E}=C_{E}(z)$ si et seulement s'il existe des entiers $r_{1}, \ldots, r_{n}$ non tous nuls et un élément $h \in K^{*}$ tels que $a_{1}^{r_{1}} \cdots a_{n}^{r_{n}}=\sigma_{q}(h) / h$;

(ii) les fonctions $f_{1}, \ldots, f_{n}$ vérifient une relation algébro-différentielle à coefficients dans $K_{E}$ si et seulement si les diviseurs elliptiques des $a_{i}$ sont $\mathbb{Z}$-linéairement dépendants.

Remarque 3.7. On peut preciser les cas intermédiaires de la facon suivante. Si les diviseurs elliptiques sont liés, on a d'après le Lemme 3.5 une relation non triviale $\prod_{i} a_{i}^{r_{i}}=\mu z^{r} \sigma_{q}(h) /(h)$, où $r_{i}, r \in \mathbb{Z}, \mu \in$ $\mathbb{C}^{*}, h \in K^{*}$. Le cas $\mu=1, r=0$ est couvert par (i). En utilisant les fonctions classiques de la théorie des $q$-différences déduites de la fonction $\theta$ (voir [Sau04, vdPS97]), on montre aisément que dans le cas contraire, l'ordre minimal des relations différentielles satisfaites par les $f_{i}$ sur $K_{E}$ est majoré par 2 , et vaut 1 si $r=0$. De plus, l'ordre 2 est effectivement atteint par la fonction $\theta$. Voir [Har0, Har06].

${ }^{2}$ Pour $N \leqslant 2$, on peut étudier le radical unipotent du groupe de Galois de l'extension $\tilde{\mathcal{M}}(N)$ elle-même à partir des méthodes de [BS99], [Ber01], et [Har0]; mais ces méthodes sont insuffisantes dès que $N \geqslant 3$. 


\section{HARDOUin}

Démonstration du Théorème 3.6. La première partie du Théorème 3.6 est un corollaire immédiat du Lemme 2.5 et des Propositions 2.2 et $2.3 \mathrm{du} \S 2$. Pour établir la seconde, il nous reste à vérifier l'énoncé suivant, dont on trouvera une preuve plus condensée en Appendice A.

Lemme 3.8. Les fonctions $\partial^{j}\left(\partial\left(a_{i}\right) / a_{i}\right)(i=1, \ldots, n, j \in \mathbb{N})$ sont linéairement dépendantes sur $\mathbb{C}$ modulo $\left(\sigma_{q}-\mathrm{id}\right)(\mathbb{C}(z))$ si et seulement si les diviseurs elliptiques $\operatorname{div}_{E}\left(a_{1}\right), \ldots, \operatorname{div}_{E}\left(a_{n}\right)$ sont linéairement dépendants sur $\mathbb{Z}$.

Démonstration. De la définition des diviseurs elliptiques et de la relation $\sigma_{q} \partial=\partial \sigma_{q}$, on déduit que les deux conditions ne dépendent que de la classe des $a_{i}$ modulo $\left\{\sigma_{q}(h) / h, h \in K^{*}\right\}$. Sans perte de généralité, on peut donc supposer que les $a_{i}$ sont standards. On fixe, une fois pour toutes, une collection $S$ de représentants de $\mathbb{C}^{*} / q^{\mathbb{Z}}$ dans $\mathbb{C}^{*}$, notée $S=\left\{\alpha \in \mathbb{C}^{*}\right\}$. On peut alors écrire les $a_{i}$ sous la forme

$$
a_{i}=\mu_{i} z^{r_{i}} \prod_{\alpha \in S}\left(z-q^{n_{i, \alpha}} \alpha\right)^{\beta_{i, \alpha}}
$$

avec $r_{i}, n_{i, \alpha}, \beta_{i, \alpha} \in \mathbb{Z}$, où l'on choisit $n_{i \alpha}$ arbitrairement si $\beta_{i, \alpha}=0$.

Supposons que les diviseurs elliptiques des $a_{i}$ sont linéairement dépendants sur $\mathbb{Z}$, et soient $l_{1}, \ldots, l_{n}$ des entiers non tous nuls tels que

$$
l_{1} \operatorname{div}_{E}\left(a_{1}\right)+l_{2} \operatorname{div}_{E}\left(a_{2}\right)+\cdots+l_{n} \operatorname{div}_{E}\left(a_{n}\right)=0
$$

dans $\operatorname{Div}\left(\mathbb{C}^{*} / q^{\mathbb{Z}}\right)$. On déduit de $(10)$ et du Lemme 3.5 qu'il existe $r \in \mathbb{Z}, \mu \in \mathbb{C}^{*}$ et $h \in K^{*}$ tels que: $\prod_{i} a_{i}^{l_{i}}=\mu z^{r} \sigma_{q}(h) /(h)$. En dérivant logarithmiquement l'équation précédente, et en dérivant une seconde fois on obtient une liaison sur $\mathbb{C}$ entre les fonctions $\partial^{j}\left(\partial\left(a_{i}\right) / a_{i}\right) i=1, \ldots, n, j \in \mathbb{N}$ (et même: $j=0,1,2)$ modulo $\left(\sigma_{q}-\mathrm{id}\right)(\mathbb{C}(z))$.

Réciproquement, supposons qu'il existe une relation de dépendance, qu'on peut choisir d'ordre $N$ minimal relativement à $j$, liant les $\partial^{j}\left(\partial\left(a_{i}\right) / a_{i}\right)(i=1, \ldots, n, j \in \mathbb{N})$ modulo $\left(\sigma_{q}-\mathrm{id}\right)(\mathbb{C}(z))$ :

$$
\sum_{i=1}^{n} \sum_{j=0}^{N} \lambda_{j}^{i} \partial^{j}\left(\partial a_{i} / a_{i}\right)=\sigma_{q}(f)-f
$$

où les $\lambda_{j}^{i}$ sont des nombres complexes, l'un des $\lambda_{N}^{i}$ est non nul, et $f$ appartient à $\mathbb{C}(z)$.

On va montrer que, pour tout $\alpha \in S$,

$$
\sum_{i=1}^{n} \lambda_{N}^{i} \beta_{i, \alpha}=0
$$

On déduit des relations (12) que les vecteurs

$$
\left(\begin{array}{c}
\beta_{1, \alpha} \\
\vdots \\
\beta_{n, \alpha}
\end{array}\right), \quad \alpha \in S
$$

de $\mathbb{Z}^{n}$ sont liés sur $\mathbb{C}$ et donc sur $\mathbb{Z}$. Par conséquent, ces relations valent avec des coefficients $\lambda_{N}^{i}$ entiers. Dans ces conditions,

$$
\sum_{i=1}^{n} \lambda_{N}^{i} \operatorname{div}_{E}\left(a_{i}\right)=\sum_{i=1}^{n} \lambda_{N}^{i}\left(\sum_{\alpha \in S} \beta_{i, \alpha}(\alpha)\right)=\sum_{\alpha \in S}\left(\sum_{i=1}^{n} \lambda_{N}^{i} \beta_{i, \alpha}\right)(\alpha)=0,
$$

et les diviseurs elliptiques des $a_{i}$ sont bien linéairement dépendants sur $\mathbb{Z}$.

Pour vérifier (12), écrivons la décomposition en éléments simples de $f$ sous la forme

$$
f(z)=\sum_{m=0}^{M} \xi_{m} z^{m}+\sum_{l=1}^{p} \sum_{r=1}^{\gamma_{l}} \frac{\nu_{r}\left(d_{l}\right)}{\left(z-d_{l}\right)^{r}}
$$




\section{HyPERTRANSCENDANCE DES SYSTÈMES AUX DIFFÉRENCES DIAGONAUX}

et reprenons les expressions $a_{i}=\mu_{i} z^{r_{i}} \prod_{\alpha \in S}\left(z-q^{n_{i, \alpha}} \alpha\right)^{\beta_{i, \alpha}}$ données par la formule (9); dans la suite, pour alléger les notations, on posera $b_{i, \alpha}=q^{n_{i, \alpha}} \alpha$ pour tout $i=1, \ldots, n, \alpha \in S$. Dans ces conditions, (11) s'écrit

$$
\sum_{i=1}^{n} \sum_{j=0}^{N} \lambda_{j}^{i} \partial^{j}\left(\partial a_{i} / a_{i}\right)=\sum_{m}\left(q^{m} \xi_{m}-\xi_{m}\right) z^{m}+\sum_{l} \sum_{r}\left(\frac{q^{-r} \cdot \nu_{r}\left(d_{l}\right)}{\left(z-d_{l} / q\right)^{r}}-\frac{\nu_{r}\left(d_{l}\right)}{\left(z-d_{l}\right)^{r}}\right) .
$$

Une récurrence aisée montre que pour tout entier $j \geqslant 0$ :

$$
\partial^{j}\left(\partial a_{i} / a_{i}\right)=\sum_{\alpha \in S} \frac{\beta_{i, \alpha}\left(b_{i, \alpha}\right)^{j+1}(-1)^{j} j !}{\left(z-b_{i, \alpha}\right)^{j+1}}+\text { des termes polaires d'ordre } \leqslant j .
$$

Fixons un élément $\alpha$ de $S$, et notons $I_{\alpha}$ l'ensemble des indices $\left\{i \in\{1, \ldots, n\}, \beta_{i, \alpha} \neq 0, \lambda_{N}^{i} \neq 0\right\}$. Remarquons tout d'abord que si $I_{\alpha}$ est vide, la relation (12) est vérifiée car, par définition de $I_{\alpha}$, on a alors $\beta_{i, \alpha}=0$ ou $\lambda_{N}^{i}=0$.

Supposons maintenant $I_{\alpha}$ réduit à un seul élément, disons $i_{0}$, et posons $b_{i_{0}}=b_{i_{0}, \alpha} \neq 0$. Compte tenu des écritures précédentes et de l'unicité de la décomposition en éléments simples sur $\mathbb{C}(z)$, on en déduit que $b_{i_{0}}$ doit être un pôle d'ordre $N+1$ de $\sigma_{q}(f)-f=\sigma_{q}(h)-h$ où l'on a posé $h=-\sigma_{q}^{-1}(f)$. Ainsi $b_{i_{0}}$ est un pôle d'ordre au au moins $N+1$ de $f$ ou de $h$. On peut donc supposer, quitte à échanger $f$ et $h$, que $b_{i_{0}}$ est pôle d'ordre au au moins $N+1$ de $f$. Soit désormais $n_{0}$ l'entier positif ou nul maximal tel que $q^{-n_{0}} b_{i_{0}}$ soit un pôle d'ordre au moins $N+1$ de $f$. Comme $q^{-\left(n_{0}+1\right)} b_{i_{0}}$ est un pôle d'ordre au moins $N+1$ de $\sigma_{q}(f)$, mais pas de $\sigma_{q}(f)-f$ (car, $a_{i_{0}}$ étant standard et $I_{\alpha}$ étant réduit à un élément, le membre de gauche de l'équation (13) ne possède qu'un pôle d'ordre au moins $N+1$ congru à $b_{i_{0}}$ modulo $\left.q^{\mathbb{Z}}\right)$, on en déduit que $q^{-\left(n_{0}+1\right)} b_{i_{0}}$ doit apparaitre comme pôle d'ordre au moins $N+1$ de $f$. Or ceci est absurde par maximalité de $n_{0}$.

On peut donc désormais supposer que $I_{\alpha}$ contient au moins deux éléments. On note $n_{1}<n_{2}<$ $\cdots<n_{t}$ les valeurs distinctes, ordonnées, prises par les $\left(n_{i, \alpha}\right), i \in I_{\alpha}$. Pour tout $l=1, \ldots, t$, on pose $I_{\alpha, n_{l}}=\left\{i \in I_{\alpha}\right.$, tels que $\left.n_{i, \alpha}=n_{l}\right\}$.

En identifiant la partie polaire d'ordre $N+1$ en la spirale $\alpha q^{\mathbb{Z}}$ du membre de gauche de l'équation (13) à celle de $\sigma_{q}(f)-f$, on obtient la relation suivante:

$$
\sum_{l=1}^{t} \sum_{i \in I_{\alpha, n_{l}}} \frac{\beta_{i, \alpha} \lambda_{N}^{i}\left(\alpha q^{n_{l}}\right)^{N+1}(-1)^{N} N !}{\left(z-\alpha q^{n_{l}}\right)^{N+1}}=\sum_{k \in \mathbb{Z}} \frac{\nu_{k+1} q^{-N-1}-\nu_{k}}{\left(z-q^{k} \alpha\right)^{N+1}},
$$

où on a posé $\nu_{k}=\nu_{N+1}\left(q^{k} \alpha\right)$ pour tout entier rationnel $k$. Par conséquent,

$$
\begin{gathered}
\forall m \notin\left\{n_{1}, \ldots, n_{t}\right\}, \nu_{m+1} q^{-N-1}-\nu_{m}=0, \\
\forall l=1, \ldots, t, \nu_{n_{l}+1} q^{-N-1}-\nu_{n_{l}}=\sum_{i \in I_{\alpha, n_{l}}} \lambda_{N}^{i} \beta_{i, \alpha}\left(\alpha q^{n_{l}}\right)^{N+1}(-1)^{N} N ! .
\end{gathered}
$$

L'ensemble des entiers $m$ tels que $\nu_{m}$ soit non nul est fini. La relation (15) entraîne que

(1) $\nu_{n_{1}}=\nu_{n_{t+1}}=0$;

$$
\forall l=1, \ldots, t-1, \prod_{m=n_{l}+1}^{n_{l+1}-1} \frac{\nu_{m+1}}{\nu_{m}}=\prod_{m=n_{l}+1}^{n_{l+1}-1} q^{N+1} .
$$

De l'équation (17) on tire les relations suivantes

$$
\forall l=1, \ldots, t-1, \nu_{n_{l+1}}=\nu_{n_{l}+1} q^{\left(n_{l+1}-n_{l}-1\right)(N+1)} .
$$




\section{HARDOUin}

En reportant dans (16), on en déduit que pour tout $l=1, \ldots, t-1$ :

$$
\nu_{n_{l+1}} q^{-n_{l+1}(N+1)}-\nu_{n_{l}} q^{-n_{l}(N+1)}=\sum_{i \in I_{\alpha, n_{l}}} \lambda_{N}^{i} \beta_{i, \alpha} \alpha^{N+1}(-1)^{N} N !
$$

et

$$
-\nu_{n_{t}} q^{-n_{t}(N+1)}=\sum_{i \in I_{\alpha, n_{t}}} \lambda_{N}^{i} \beta_{i, \alpha} \alpha^{N+1}(-1)^{N} N !
$$

En sommant toutes ces équations, on obtient

$$
\sum_{l=1}^{t} \sum_{i \in I_{\alpha, n_{l}}} \beta_{i, \alpha} \lambda_{N}^{i} \alpha^{N+1}(-1)^{N} N !=-\nu_{n_{1}}=0
$$

d'où $\sum_{i=1}^{n} \lambda_{N}^{i} \beta_{i, \alpha}=0$, puisque $I_{\alpha}=\bigcup_{l=1}^{t} I_{\alpha, n_{l}}$. Le Lemme 3.8 est donc démontré.

Ceci conclut la preuve des Théorèmes 3.6 et 1.1 .

\section{Hypertranscendance des solutions d'équations aux $\tau$-différences}

\section{1 Énoncé des résultats}

Nous montrons dans cette dernière partie que les résultats précédents s'étendent sans changement majeur à l'étude des $\tau$-différences. Précisons-en tout d'abord le cadre.

Soit $\tau \in \mathbb{C}$ un nombre complexe non nul. On désigne par $K=\mathbb{C}(z)$ le corps des fractions rationelles à coefficients complexes, par $F=\operatorname{Mer}(\mathbb{C})$ le corps des fonctions méromorphes sur $\mathbb{C}$ et par $\sigma_{\tau}$ l'automorphisme de $F$ qui à $f(z) \in F$ associe $f(z+\tau)$. On note $C_{\tau}$ le sous-corps de $F$ formé par les fonctions $\tau$-périodiques (c'est-à-dire les éléments fixés par $\sigma_{\tau}$ ), et $K_{\tau}=C_{\tau}(z)$ le compositum de $C_{\tau}$ et de $K$ dans $F$. Les corps $K, F$ et $K_{\tau}$ sont des corps aux différences relativement à $\sigma_{\tau}$, admettant respectivement pour corps des $\sigma_{\tau}$-constantes $\mathbb{C}, C_{\tau}$ et $C_{\tau}$.

L'automorphisme $\sigma_{\tau}$ et la dérivation $\partial=d / d z$ munissent le corps $F=\operatorname{Mer}(\mathbb{C})$ et ses sous-corps $K_{\tau}$ et $K$ de structures de corps aux différences différentiels, puisque $\sigma_{\tau} \partial=\partial \sigma_{\tau}$. Ces trois corps admettent $\mathbb{C}$ comme corps de constantes différentielles.

DÉfinition 4.1. Soit $a \in \mathbb{C}(z)^{*}$. On note $\operatorname{div}_{\tau}(a)$ l'image de la partie première à $\{\infty\}$ du diviseur de $a$ par l'application naturelle de $\operatorname{Div}(\mathbb{C})$ dans $\operatorname{Div}(\mathbb{C} / \tau \mathbb{Z})$ et on l'appelle diviseur périodique de $a$.

Voici, dans ces conditions, le $\tau$-analogue des Théorèmes 1.1 et 3.6.

ThÉORÈme 4.2. Soient $a_{1}, \ldots, a_{n}$ des éléments non nuls de $K=\mathbb{C}(z)$ et $\tau$ un nombre complexe non nul. Pour tout $i=1, \ldots, n$, soit $f_{i} \neq 0$ une solution méromorphe sur $\mathbb{C}$ de l'équation aux $\tau$-différences $\sigma_{\tau}\left(f_{i}\right)=a_{i} f_{i}$. Alors:

(i) les fonctions $f_{1}, \ldots, f_{n}$ sont algébriquement dépendantes sur $K_{\tau}$ si et seulement s'il existe des entiers $r_{1}, \ldots, r_{n}$ non tous nuls et un élément $h \in K^{*}$ tels que $a_{1}^{r_{1}} \cdots a_{n}^{r_{n}}=\sigma_{\tau}(h) / h$;

(ii) les fonctions $f_{1}, \ldots, f_{n}$ vérifient une relation algébro-différentielle à coefficients dans $K_{\tau}$ si et seulement si les diviseurs périodiques des $a_{i}$ sont $\mathbb{Z}$-linéairement dépendants.

Les démonstrations de ces énoncés relèvent des mêmes procédés que pour les $q$-différences. On n'en explicitera donc pas les détails. Signalons seulement que le point (0) n'est plus, contrairement aux $q$-différences, fixé par l'opérateur $\sigma_{\tau}$. C'est la raison pour laquelle la partie supportée par 0 des diviseurs de $a_{i}$ ne joue ici plus de rôle particulier. Plus précisément, l'analogue du Lemme 3.5 s'écrit maintenant. 


\section{HyPERTRANSCENDANCE DES SYSTÈMES AUX DIFFÉRENCES DIAGONAUX}

Lemme 4.3. Soient $a \in K^{*}$. Alors $\operatorname{div}_{\tau}(a)=0$ si et seulement s'il existe un nombre complexe $\mu \in \mathbb{C}^{*}$ et $h \in K^{*}$ tel que $a=\mu \sigma_{\tau}(h) / h$.

Pour la même raison, l'ordre minimal des relations différentielles éventuelles liant des solutions $f_{i}$ d'équations aux $\tau$-différences est majoré par 1 , et non à 2 comme dans le cas des $q$-différences (voir Remarque 3.7).

\subsection{Application à la fonction $\Gamma$}

Dans cette application $\tau=1$, et on fixe des nombres complexes $\alpha_{1}, \ldots, \alpha_{k}$ ainsi que deux entiers $n, m \geqslant 2$. La relation de distribution

$$
\Gamma(z) \Gamma\left(z+\frac{1}{n}\right) \cdots \Gamma\left(z+\frac{n-1}{n}\right)=(2 \pi)^{(n-1) / 2} n^{1 / 2-n z} \Gamma(n z)
$$

(et l'équation différentielle $\left.(d / d z) m^{z}=(\log m) m^{z}\right)$ montrent que si $k=n$ et si $\alpha_{i}$ est congru à $i / n$ modulo $\mathbb{Z}$ pour tout $i$, alors les fonctions $\Gamma\left(z+\alpha_{1}\right), \ldots, \Gamma\left(z+\alpha_{n}\right), \Gamma(n z)$ sont hyperalgébriquement dépendantes sur $K$; si de plus $m$ et $n$ sont multiplicativement dépendants, alors ces fonctions ainsi que $m^{z}$ sont algébriquement dépendantes sur $K$. Inversement on a Corollaire 4.4.

Corollaire 4.4. Soient $\alpha_{1}, \ldots, \alpha_{k}$ des éléments de $\mathbb{C}$ et $n_{1}, \ldots, n_{h}, m$ des entiers $\geqslant 2$. On suppose que les diviseurs périodiques $\left(\bar{\alpha}_{i}\right), i=1, \ldots, k,\left(\sum_{l=0}^{n_{j}-1}\left(\bar{l} / n_{j}\right)\right), j=1, \ldots, h$, sont $\mathbb{Z}$-linéairement indépendants. Alors:

(1) les fonctions $\Gamma\left(z+\alpha_{1}\right), \ldots, \Gamma\left(z+\alpha_{k}\right), \Gamma\left(n_{1} z\right), \ldots, \Gamma\left(n_{h} z\right)$ et $m^{z}$ sont algébriquement indépendantes $\operatorname{sur} K_{\tau}$;

(2) les fonctions $\Gamma\left(z+\alpha_{i}\right), \Gamma\left(n_{j} z\right), i=1, \ldots, k, j=1, \ldots, h$, sont hyperalgébriquement indépendantes sur $K_{\tau}$.

On a ici $\sigma_{\tau}\left(\Gamma\left(z+\alpha_{i}\right)\right)=\Gamma\left(z+\alpha_{i}+1\right)=\left(z+\alpha_{i}\right) \Gamma\left(z+\alpha_{i}\right)$ et $\sigma_{\tau}\left(\Gamma\left(n_{j} z\right)=\Gamma\left(n_{j} z+n_{j}\right)=\right.$ $\prod_{l=0}^{n_{j}-1}\left(n_{j} z+l\right) \Gamma\left(n_{j} z\right)$. Il en résulte que pour ce système, les diviseurs périodiques sont exactement $\left(\bar{\alpha}_{i}\right), i=1, \ldots, k,\left(\sum_{l=0}^{n_{j}-1}\left(\bar{l} / n_{j}\right)\right), j=1, \ldots, h$. En appliquant le Théorème 4.2 , on obtient la conclusion désirée.

\section{Appendice A. Une preuve plus concise du Théorème 1.1 par van der Put [vdP07]}

On fixe dans cet appendice un corps algébriquement clos de caractéristique nulle $\tilde{C}$ qui, en fin de preuve, sera choisi égal à la clôture algébrique du corps $C_{E}=\mathbf{C}(E)$ apparaissant dans l'article. Soient $q$ un élément de $\tilde{C}^{*}$ non racine de l'unité, $\tilde{K}=\tilde{C}(z)$ le corps des fonctions rationnelles sur $\tilde{C}$, et $\sigma=\sigma_{q}$ l'automorphisme de $\tilde{K}$ tel que $\sigma(f(z))=f(q z)$. La dérivation $\partial=z d / d z$ de $\tilde{K}$ commute avec $\sigma$.

Un diviseur sur le groupe $B=\tilde{C}^{*} / q^{\mathbb{Z}}$ est une application de $B$ dans $\mathbb{Z}$ (ou plus généralement dans $\tilde{C}$ ou dans toute extension de $\tilde{C}$ ) de support fini. À tout élément $a$ de $\tilde{K}^{*}$, on associe le diviseur $D_{a}: B \rightarrow \mathbb{Z}$ sur $B$ défini par

$$
D_{a}(b)=\sum_{\alpha \in \tilde{C}^{*}, \operatorname{pr}(\alpha)=b} \operatorname{ord}_{\alpha}(a),
$$

oú $\mathrm{pr}: \tilde{C}^{*} \rightarrow B$ est la projection canonique.

Soient $a_{1}, \ldots, a_{n}$ des éléments de $\tilde{K}^{*}$ et $N$ un entier naturel positif. On considère le système d'équations aux différences

$$
\sigma\left(Y_{i}\right)=a_{i} Y_{i}, \sigma\left(Z_{i, j}\right)=Z_{i, j}+\partial^{j}\left(\frac{\partial a_{i}}{a_{i}}\right), \quad i=1, \ldots, n, j=0, \ldots, N-1 .
$$




\section{HARdouin}

Puisque le corps de $\sigma$-constantes $\tilde{C}$ de $\tilde{K}$ est algébriquement clos, on dispose pour ce système (voir [vdPS97, Theorem 1.13]):

(a) d'un anneau de Picard-Vessiot $R$, unique à isomorphisme près, qu'on peut décrire comme l'anneau quotient

$$
R=\tilde{K}\left[\left\{Y_{i}, Y_{i}^{-1}, Z_{i, j}\right\}, \quad 1 \leqslant i \leqslant n, 0 \leqslant j \leqslant N-1\right] / \tilde{I},
$$

oú $\sigma$ est défini par $\sigma\left(Y_{i}\right)=a_{i} Y_{i}, \sigma\left(Z_{i, j}\right)=Z_{i, j}+\partial^{j}\left(\partial a_{i} / a_{i}\right)$ et $\tilde{I}$ est un idéal de l'anneau $\tilde{K}\left[\left\{Y_{i}, Y_{i}^{-1}, Z_{i, j}\right\}, 1 \leqslant i \leqslant n, 0 \leqslant j \leqslant N-1\right]$ maximal parmi les idéaux stables sous $\sigma$;

(b) du groupe de Galois aux différences $\Gamma=A u t_{\sigma}(R / \tilde{K})$ de $(*)$; il s'identifie à un groupe algébrique sur $\tilde{C}$, et vérifie $R^{\Gamma}=\tilde{K}$, et $\operatorname{deg} \operatorname{tr}(R / \tilde{K})=\operatorname{dim}_{\tilde{C}} \Gamma$.

ThÉorème A.1. Pour $i=1, \ldots, n$, soit $D_{i}=D_{a_{i}}$ le diviseur sur $B$ associé à $a_{i}$. Supposons que les $D_{i}$ sont $\mathbb{Z}$-linéairement indépendants. Alors le groupe de Galois aux différences $\Gamma$ du système $(*)$ est isomorphe $\mathbb{G}_{m}^{n} \times \mathbb{G}_{a}^{n N}$, et l'idéal $\tilde{I}$ est nul.

Démonstration. De la conclusion sur le groupe $\Gamma$, on déduit que

$$
\operatorname{degtr}_{\tilde{K}} R=\operatorname{dim}_{\tilde{C}} \Gamma=n(N+1)=\operatorname{degtr}_{\tilde{K}} \tilde{K}\left[\left\{Y_{i}, Y_{i}^{-1}, Z_{i, j}\right\}, 1 \leqslant i \leqslant n, 0 \leqslant j \leqslant N-1\right],
$$

de sorte que l'idéal $\tilde{I}$ est bien nul. Il reste à calculer $\Gamma$.

Comme dans la preuve de la Proposition 2.7, le groupe de Galois $\Gamma$ du système $(*)$ est un sousgroupe algébrique de $\mathbb{G}_{m}^{n} \times \mathbb{G}_{a}^{n N}$, donc de la forme $\Gamma_{1} \times \Gamma_{2}$ avec $\Gamma_{1} \subset \mathbb{G}_{m}^{n}$ et $\Gamma_{2} \subset \mathbb{G}_{a}^{n N}$. À l'instar des Propositions 2.1 et 2.2 (dont la relation $R^{\Gamma}=\tilde{K}$ permet ici de simplifier les preuves), on vérifie aisément les énoncés suivants:

(a) si $\Gamma_{1} \subsetneq \mathbb{G}_{m}^{n}$, alors il existe des entiers rationnels $\left(m_{1}, \ldots, m_{n}\right)$ non tous nuls tels que l'équation $\sigma(Y)=a_{1}^{m_{1}} \cdots a_{n}^{m_{n}} Y$ ait une solution non triviale $f$ dans $\tilde{K}$

(b) si $\Gamma_{2} \subsetneq \mathbb{G}_{a}^{n N}$, alors il existe des éléments $\gamma_{i, j} \in \tilde{C}$, non tous nuls, tels que l'équation $\sigma(Z)=$ $Z+\sum_{i, j} \gamma_{i, j} \partial^{j}\left(\partial a_{i} / a_{i}\right)$ ait une solution $y$ dans $\tilde{K}$.

Considérons le point (a). L'égalité $\sigma(f) / f=a_{1}^{m_{1}} \cdots a_{n}^{m_{n}}$ implique $\sum_{i=1}^{n} m_{i} D_{i}=D_{\sigma(f)}-D_{f}=0$, en contradiction avec l'hypothèse du Théorème A.1. Ainsi, $\Gamma_{1}=\mathbb{G}_{m}^{n}$.

Considérons le point (b), dont on va montrer, comme au Lemme 3.8 du texte (où $\tilde{C}=\mathbf{C}$ ), que la conclusion contredit à nouveau l'hypothèse faite sur les diviseurs $D_{i}$. Pour caractériser les éléments de $\tilde{K}$ de la forme $\sigma(y)-y$, où $y \in \tilde{K}$, les notations suivantes seront utiles. Puisque $\tilde{C}$ est algébriquement clos, tout élément $y$ de $\tilde{K}$ se décompose de façon unique sous la forme

$$
P(z)+\sum_{m \geqslant 1} \frac{c(m)}{z^{m}}+\sum_{b \in B, m \geqslant 1} \sum_{\beta \in b} \frac{c(m, \beta)}{(z-\beta)^{m}},
$$

oú $P(z)$ est un polynôme. On associe alors à $y$ l'élément $e(y)=P(0)$ de $\tilde{C}$, et, pour tout entier $m \geqslant 1$, le diviseur $\operatorname{div}(m, y)$ sur $B$, à valeurs dans $\tilde{C}$, donné par $\operatorname{div}(m, b)=\sum_{\beta \in b} \beta^{-m} c(m, \beta) ;$ on définit enfin le symbole formel

$$
S(y)=e(y)+\sum_{m \geqslant 1} \frac{\operatorname{div}(m, y)}{(1+X)^{m}} .
$$




\section{HyPERTRANSCENDANCE DES SYSTÈMES AUX DIFFÉRENCES DIAGONAUX}

Dans ces conditions, on a

$$
\begin{aligned}
\sigma(y) & =P(q z)+\sum_{m \geqslant 1} \frac{q^{-m} c(m)}{z^{m}}+\sum_{b \in B, m \geqslant 1} \sum_{\beta \in b} \frac{q^{-m} c(m, q \beta)}{(z-\beta)^{m}}, \\
\partial y & =\partial(P(z))+\sum_{m \geqslant 1} \frac{-m c(m)}{z^{m}}+\sum_{b \in B, m \geqslant 1} \sum_{\beta \in b} \frac{-m c(m, \beta)-(m-1) c(m-1, \beta) \beta}{(z-\beta)^{m}}, \\
\text { si } a & =\mu z^{m} \prod_{b \in B, \beta \in b}(z-\beta)^{m(\beta)}, \text { alors } \frac{\partial(a)}{a}=\sum m(\beta)+\frac{m}{z}+\sum_{b \in B, \beta \in b} \frac{m(\beta) \beta}{(z-\beta)} .
\end{aligned}
$$

On vérifie facilement les énoncés suivants:

(1) $e(\sigma(y))=e(y), \operatorname{div}(m, \sigma y)=\operatorname{div}(m, y)$ et $S(\sigma y)=S(y)$;

(2) soit $h$ un élément de $\tilde{K}$, l'équation $\sigma(y)-y=h$ admet une solution $y \in \tilde{K}$ si et seulement si $S(h)=0$ (nous n'aurons d'ailleurs besoin que de la nécessité de cette condition, qui découle immédiatement du point 1$)$;

(3) $\partial y$ vérifie $e(\partial y)=0, \operatorname{div}(m, \partial y)=-m \cdot \operatorname{div}(m, y)-(m-1) \cdot \operatorname{div}(m-1, y)$ et $S(\partial y)=(2+$ $X)(d / d X) S(y)$

(4) pour $i=1, \ldots, n$, posons $a_{i}=c_{i} \prod_{b \in B} \prod_{\beta \in b}(z-\beta)^{m_{i}(\beta)}$, de sorte que le diviseur $D_{i}: B \rightarrow \mathbb{Z}$ associé $a_{i}$ vérifie $D_{i}(b)=\sum_{\beta \in b} m_{i}(\beta)$, et alors $S\left(\partial a_{i} / a_{i}\right)=\operatorname{deg} D_{i}+D_{i} /(1+X)$.

Considérons l'expression $h=\sum_{i, j} \gamma_{i, j} \partial^{j}\left(\partial a_{i} / a_{i}\right)$, où les $\gamma_{i, j}$ sont des éléments de $\tilde{C}$. Supposons, suivant le point (b), qu'il existe $y \in K$ tel que $h=\sigma(y)-y$. On va en déduire que tous les $\gamma_{i, j}$ sont nuls. D'après les formules précédentes, on a

$$
0=S(h)=\sum_{i=1}^{n} \gamma_{i, 0} \operatorname{deg} D_{i}+\sum_{i=1}^{n}\left(\sum_{0 \leqslant j \leqslant N-1} \gamma_{i, j}\left((2+X) \frac{d}{d X}\right)^{j}\left(\frac{1}{1+X}\right)\right) \cdot D_{i} .
$$

Par hypothèse, les diviseurs $D_{i}$ sont linéairement indépendants sur $\mathbb{Z}$, donc aussi sur tout anneau contenant $\mathbb{Z}$, et en particulier sur $\tilde{C}(X)$. Ainsi les fonctions rationnelles

$$
\sum_{0 \leqslant j \leqslant N-1} \gamma_{i, m}\left((2+X) \frac{d}{d X}\right)^{j}\left(\frac{1}{1+X}\right)
$$

sont nulles pour $i=1, \ldots, n$. Cela implique que les $\gamma_{i, j}$ sont nuls, et en définitive, qu'on a bien: $\Gamma_{2}=\mathbb{G}_{a}^{n N}$.

Remarque A.2 (Variante aux $\tau$-différences). Soit $\tau$ un élément non nul de $\tilde{C}$. Munissons le corps $\tilde{K}=\tilde{C}(z)$ de l'opérateur aux différences $\phi(f)(z)=f(z+\tau)$. Soient $a_{1}, \ldots, a_{n}$ des éléments de $\tilde{K}^{*}$, dont on suppose les diviseurs modulo $\mathbb{Z} \tau$ linéairement indépendants sur $\mathbb{Z}$. Considérons le système $\phi\left(Y_{i}\right)=a_{i} Y_{i}$,

$$
\phi\left(Z_{i, j}\right)=Z_{i, j}+\left(\frac{d}{d z}\right)^{j}\left(a_{i}^{-1} \frac{d a_{i}}{d z}\right)
$$

où $i=1, \ldots, n, 0 \leqslant j \leqslant N-1$. Alors le groupe de Galois aux différences de ce système est $\mathbb{G}_{m}^{n} \times \mathbb{G}_{a}^{n N}$. La preuve est similaire celle du Théorème A.1.

On peut maintenant appliquer le Théorème A.1 la situation particulière du Théorème 1.1. Soit donc $q \in \mathbb{C}$, tel que $0<|q|<1$ et soit $E$ la courbe elliptique $\mathbb{C}^{*} / q^{\mathbb{Z}}$. On note $p r: \mathbb{C}^{*} \rightarrow E$ la projection naturelle. Pour tout diviseur sur $\mathbb{C}^{*}$ (à support fini), on désigne par $\operatorname{pr}(D)$ l'image de $D$ dans le groupe des diviseurs sur $E$. Pour $a \in \mathbb{C}(z)$, le diviseur $\left.\left.\operatorname{pr}(\operatorname{div}(a))\right|_{\mathbb{C}^{*}}\right)$ est ainsi le diviseur elliptique de $a$, au sens de la Définition 3.4. Comme au $\S 3$, considérons le corps $F=\mathcal{M e r}\left(\mathbb{C}^{*}\right)$ des fonctions méromorphes sur $\mathbb{C}^{*}$, muni de l'automorphisme $\sigma$ défini par $\sigma(f)(z)=f(q z)$. Le corps 


\section{HARDOUin}

$C_{E}=\mathbb{C}(E)$ des fonctions rationnelles sur $E$ s'identifie au corps $F^{\langle\sigma\rangle}$ des fonctions méromorphes invariantes sous $\sigma$. La dérivation $\partial$ de $F$ définie par $\partial(f)=z d f / d z$ commute avec l'opérateur $\sigma$.

Corollaire A.3 (Théorème 1.1). Soient $a_{1}, \ldots, a_{n}$ des éléments de $\mathbb{C}(z)^{*}$. Pour tout $i=1, \ldots, n$, soit $f_{i}$ un élément de $F^{*}$ tel que $\sigma\left(f_{i}\right)=a_{i} f_{i}$. Supposons que les diviseurs $D_{i}=\operatorname{pr}\left(\left.\operatorname{div}\left(a_{i}\right)\right|_{\mathbb{C}^{*}}\right)$ sur $E$ soient linéairement indépendants sur $\mathbb{Z}$. Alors la famille de fonctions méromorphes $\left\{\partial^{j}\left(f_{i}\right), i=\right.$ $1, \ldots, n, j \geqslant 0\} \subset F$ est algébriquement indépendante sur le sous-corps $C_{E}(z)$ de $F$.

Démonstration. On notera que le théorème de Praagman [Pra86] rappelé au $\S 3$ assure l'existence d'une solution $f_{i} \in F^{*}$ de l'équation $\sigma(y)=a_{i} y$, et celle-ci est unique multiplication par un élément de $C_{E}^{*}$ près.

Comme au $\S 3.1$, on se ramène à étudier le système aux différences sur le sous-corps $K_{E}=C_{E}(z)$ de $F$ donné par

$$
\sigma\left(Y_{i}\right)=a_{i} Y_{i} ; \sigma\left(Z_{i, j}\right)=Z_{i, j}+\partial^{j}\left(\frac{\partial a_{i}}{a_{i}}\right) ; \quad i=1, \ldots, n, 0 \leqslant j \leqslant N-1,
$$

où $N$ est un entier positif arbitraire. L'homomorphisme de $K_{E}$-algèbres

$$
h: K_{E}\left[\left\{Y_{i}, Y_{i}^{-1}, Z_{i, j}\right\} ; i=1, \ldots, n, j=0, \ldots, N-1\right] \rightarrow F
$$

défini par $h\left(Y_{i}\right)=f_{i}$ et $h\left(Z_{i, j}\right)=\partial^{j}\left(\partial f_{i} / f_{i}\right)$ est clairement $\sigma$-invariant. Le noyau $J$ de $h$ est donc un idéal de $K_{E}\left[\left\{Y_{i}, Y_{i}^{-1}, Z_{i, j}\right\}\right]$ stable sous $\sigma$.

Soit alors $\tilde{C}$ une clôture algébrique de $C_{E}$, et $\tilde{K}$ le corps $\tilde{C}(z)$, muni du prolongement de $\sigma$ induisant l'identité sur $\tilde{C}$. L'idéal $J$ engendre dans la $\tilde{K}$-algèbre $\tilde{K}\left[\left\{Y_{i}, Y_{i}^{-1}, Z_{i, j}\right\}\right]$ un idéal $\tilde{J}$ stable sous $\sigma$, donc contenu dans l'idéal maximal $\tilde{I}$ étudié au Théorème A.1. D'après ce théorème, $\tilde{J} \subset \tilde{I}$ est nul, de sorte que $J$ l'est aussi, et ceci démontre l'indépendance algébrique des $f_{i}$ ainsi que de leurs dérivées sur le corps $K_{E}$.

\section{REMERCIEMENTS}

Je remercie D. Bertrand pour son introduction à ces questions d'hypertranscendence, M. Singer et Z. Chatzidatkis pour nos discussions communes et pour notre travail sur la définition des groupes de Galois aux différences, et J. Oesterlé pour ses éclaircissements sur les groupes unipotents. Enfin, je remercie M. van der Put de m'avoir permis d'inclure ici sa nouvelle preuve [vdP07] du Théorème 1.1.

\section{REFERENCES}

And01 Y. André, Différentielles non commutatives et théorie de Galois différentielle ou aux différences, Ann. Sci. École Norm. Sup. (4) 34, (2001), 685-739.

Ber01 D. Bertrand, Unipotent radicals of differential Galois groups and integrals of solutions of inhomogeneous equations, Math. Ann. 321 (2001), 645-666.

Ban80 S. B. Bank, Some results on hypertranscendental meromorphic functions, Monatsh. Math. 90 (1980), 267-289.

BS99 P. H. Berman and M. F. Singer, Calculating the Galois group of $L_{1}\left(L_{2}(y)\right)=0, L_{1}, L_{2}$ completely reducible operators, J. Pure Appl. Algebra 139 (1999), 3-23.

Béz00 J.-P. Bézivin, Sur les systèmes d'équations aux différences, Aequationes Math. 60 (2000), 80-98.

CHS08 Z. Chatzidatkis, C. Hardouin and M. F. Singer, On the definitions of difference Galois groups, in Proc. conf. model theory and applications, 2005 (Cambridge University Press, Cambridge, 2008), à paraître.

Del90 P. Deligne, Catégories tannakiennes, Progress in Mathematics, vol. 87 (Birkhäuser, Boston, 1990), $111-195$. 


\section{HyPERTRANSCENDANCE DES SYSTÈMES AUX DIFFÉRENCES DIAGONAUX}

DRSZ03 L. Di Vizio, J. P. Ramis, J. Sauloy and C. Zhang. Équations aux q-différences, Gazette des Mathématiciens 96 (2003), 20-49.

Har0 C. Hardouin, Structure galoisienne des extensions itérées de modules différentiels, Thèse de doctorat de l'université Paris VI, accessible sur http://www.math.jussieu.fr/hardouin/thesera6.pdf.

Har06 C. Hardouin, Hypertranscendance et groupes de Galois aux différences, Preprint (2006), arXiv math.QA/0609646.

Ish98 K. Ishizaki, Hypertranscendency of meromorphic solutions of a linear functional equation, Aequationes Math. 56 (1998), 271-283.

Kat90 N. M. Katz, Exponential sums and differential equations, Annals of Mathematics Studies, vol. 124 (Princeton University Press, Princeton, NJ, 1990).

Ovc06 A. Ovchinnikov, Tannakian categories, linear differential algebraic groups, and parametrized linear differential equations, Preprint, NCSU (2006).

Pra86 C. Praagman, Fundamental solutions for meromorphic linear difference equations in the complex plane, and related problems, J. reine angew. Math. 369 (1986), 101-109.

vdP07 M. van der Put, Manuscrit (2007).

vdPS97 M. van der Put and M. F. Singer, Galois theory of difference equations, Lecture Notes in Mathematics, vol. 1666 (Springer, Berlin, 1997).

Ram92 J.-P. Ramis, About the growth of entire functions solutions of linear algebraic q-difference equations, Ann. Fac. Sci. Toulouse Math. (6) 1 (1992), 53-94.

Sau04 J. Sauloy, Galois theory of Fuchsian q-difference equations, Ann. Sci. École Norm. Sup. (4) 36, (2004), 925-968.

Spr98 T. A. Springer, Linear algebraic groups, Progress in Mathematics, vol. 9 (Birkhäuser, Boston, 1998).

Charlotte Hardouin charlotte.hardouin@iwr.uni-heidelberg.de

Interdisziplinäres Zentrum für Wissenschaftliches Rechnen (IWR), Universität Heidelberg, Im Neuenheimer Feld 368, 69120 Heidelberg, Germany 